\title{
Balkanologie
}

Balkanologie Revue d'études pluridisciplinaires

Vol. VIII, $n^{\circ} 2$ | 2004

Volume VIII Numéro 2

\section{Réinterprétation du passé et imaginaire urbain}

Patrimoine architectural, politique culturelle et peinture figurative à Plovdiv, Bulgarie

Re-Interpretation of Past and urban imaginary in Plovdiv

Krassimira Krastanova et Michel Rautenberg

\section{OpenEdition}

Journals

Édition électronique

URL : http://journals.openedition.org/balkanologie/535

DOI : $10.4000 /$ balkanologie. 535

ISSN : 1965-0582

Éditeur

Association française d'études sur les Balkans (Afebalk)

Édition imprimée

Date de publication : 1 décembre 2004

ISSN : 1279-7952

Référence électronique

Krassimira Krastanova et Michel Rautenberg, " Réinterprétation du passé et imaginaire urbain », Balkanologie [En ligne], Vol. VIII, n² 2 | 2004, mis en ligne le 21 janvier 2010, consulté le 17 décembre 2020. URL : http://journals.openedition.org/balkanologie/535 ; DOI : https://doi.org/10.4000/ balkanologie. 535 


\title{
RÉINTERPRÉTATION DU PASSÉ ET IMAGINAIRE URBAIN. PATRIMOINE ARCHITECTURAL, POLITIQUE CULTURELLE ET PEINTURE FIGURATIVE À PLOVDIV, BULGARIE ${ }^{1}$
}

\author{
Krassimira Krastanova* \\ Michel Rautenberg**
}

En Bulgarie comme partout dans l'est de l'Europe, la " naissance de la nation " va se construire sur la chute des empires, au moment du passage à la modernité politique. Celle-ci, favorisée par le développement des moyens de communication que représente la presse et par l'urbanisation consécutive à l'intensification des échanges commerciaux et à l'essor de l'industrie ${ }^{2}$, entraîne l'affaiblissement des solidarités communautaires et l'accentuation de la différentiation sociale entre nationaux. La culture apparait comme un nouveau ciment identitaire, la matière première d'un " imaginaire national " 3 que chaque citoyen sera invité à partager.

Plovdiv, s'appuyant sur son dynamisme économique et culturel, prend au $\mathrm{XIX}^{\mathrm{e}}$ siècle une place importante dans la lutte pour l'indépendance et devient l'un des hauts lieux de la revendication nationale. Ville d'histoire, ville de culture et de commerce, elle possède une tradition urbaine bien réelle au moment de la libération du " joug ottoman ", elle est également une véritable capitale régionale située au cœur d'un vaste arrière pays rural, la Thrace, non loin des montagnes des Rhodopes. Mais elle est aussi ouverte sur le monde, sa

\footnotetext{
- Chercheure à l'Institut de Folklore de l'Académie des sciences de Bulgarie, krassi_krastanova@evrocom.net

* Professeur à l'université des sciences et technologie de Lille, Centre lillois d'études et de recherches sociologiques et économiques (UMR 8019 du CNRS), mrautenberg@infonie.fr

\begin{abstract}
${ }^{1}$ Les enquêtes sur lesquelles nous nous appuyons ont été conduites entre février 2002 et octobre 2003 auprès d'artistes, de responsables culturels et de fonctionnaires ayant travaille pour la ville de Plovdiv depuis les années 1950. Ces premiers résultats ont été présentés à Berlin, à la Fifth European Social Science History Conference, 24-26 mars 2004, et à Marseille, colloque SIEF-ADAM, Entre Autres, rencontres et conflits en Méditerranée, 26-30 avril 2004.
\end{abstract}

${ }^{2}$ Gellner (Ernst), Nations et nationalismes, Paris : Payot, 1999.

${ }^{3}$ Anderson (Benedict), Imagined Communities, London : Verso / New Left Books, 1983. 
bourgeoisie voyage et fait affaire avec l'Europe, la Méditerranée, l'Orient. Dès la fin du XIX ${ }^{e}$ siècle, le pouvoir municipal aura le projet d'en faire une ville moderne et de maîtriser son développement. Par ailleurs sa culture, sa " personnalité urbaine " pour reprendre l'expression de Redfield et Ranger4, relève à la fois des influences de son arrière pays, elle est une " ville-capitale " qui draine les forces vives des régions alentours; et de la diversité de ses habitants qui impriment à son activité l'hétérogénéité de leurs traditions culturelles. Elle est un lieu de confrontation et de mélange tout en faisant la synthèse de la vitalité intellectuelle de la région alentours 5 .

Dans cet article nous nous proposons de montrer comment le patrimoine architectural et la peinture ont contribué à alimenter la construction d'un imaginaire urbain singulier, trouvant ses sources dans cette histoire locale particulière, imaginaire qui va lui-même influencer les politiques de conservation et les choix esthétiques des artistes. Pour cela nous mobiliserons les thèses, désormais classiques, développées par les historiens et anthropologues de la construction des nations, et les problématiques de l'anthropologie urbaine selon lesquelles la modernisation des villes s'est faite par la mise en place d'organisations favorisant le contrôle social, dans un contexte de gestion des tensions entre les logiques culturelles fusionnelles et les logiques culturelles hétérogènes qui les traversent. Plus précisément, ce que nous tenterons de montrer dans cet article, c'est comment Plovdiv s'est parée des atours d'une singularité urbaine construite par la communication, dans le sens où l'entend Louis Wirth ${ }^{6}$, faite autour de son patrimoine architectural et de la présence d'une importante communauté d'artistes?. Le paradigme patrimonial et artistique, appuyant le propos sur l'identité nationale, s'est développé au détriment d'autres spécificités historiques et sociologiques de la ville, également riche d'une longue histoire intercommunautaire entre Bulgares, Turcs, Arméniens, Tsiganes, Juifs. Ajoutons que dans cette histoire culturelle de Plovdiv deux hommes, au moins, semblent avoir joué un rôle déterminant

4 Cités par Hannerz (Ulf), Cultural Complexity. Studies in the Social Organization of Meanning, New York: Columbia University Press, 1992, p. 198.

5 Cette vitalité des traditions communautaires de Plovdiv a été savoureusement décrite par A. Wagenstein dans son roman Wagenstein (Angel), Abraham le poivrot, Paris : L'esprit des péninsules, 2002.

${ }^{6}$ Selon Louis Wirth, face à la masse urbaine et à la segmentarisation des relations entre les hommes, dans la ville moderne la communication va prendre une place éminente : " plus il y a de gens en état d'interaction plus faible est le niveau de communication (...) n et plus grande est alors la tendance à établir la communication sur ce qu'on pense être commun à tous. Wirth (Louis), "Le phénomène urbain comme mode de vie ", in Grafmeyer (Yves), Joseph (Isaac), éds., L'école de Chicago. Naissance de l'écologie urbaine, Paris : Aubier, 1979.

7 En 2002 Plovdiv a été capitale européenne de la culture. Précisons que l'association des peintres plovdiviens comprend environ 400 membres. 
en sachant utiliser les institutions publiques comme il le fallait. Le premier, architecte, en identifiant et en rationalisant la connaissance de l'architecture de la vieille ville ; le second, fonctionnaire de la ville, en promouvant parallèlement ce patrimoine réinventé et les peintres plovdiviens.

\section{LA BULGARIE}

À son indépendance, en 1879, la Bulgarie était très sensible à son héritage culturel. Le patrimoine culturel devint l'emblème de la " bulgarité " et il participa à la construction de l'identité nationale, comme en beaucoup d'autres pays européens émergeant de la dislocation des empires. Comme partout, ce patrimoine est sélectif : dans un premier temps les édifices islamiques en sont exclus et beaucoup sont détruits. Le patrimoine doit d'abord servir à exprimer les racines pré-ottomanes de la culture bulgare. Art, folklore, musique populaire, littérature orale sont mobilisés pour construire l'identité culturelle de la nation, en même temps que son histoire est écrite, sur un mode souvent hagiographique, et qu'ouvrent des musées dans lesquels sont déclinées les spécificités locales de la nation nouvelle. Dans ce contexte, Plovdiv devait rendre compte des fondements historiques du pays à travers son architecture, si typique de la période de la Renaissance bulgare ${ }^{8}$, entre la fin du XVIII ${ }^{e}$ siècle et le milieu du XIX ${ }^{\mathrm{e}}$ siècle pendant laquelle se constitue une bourgeoisie et où émerge une classe intellectuelle.

À l'époque, des voix s'élèvent déjà pour souligner les risques de l'ouverture au monde consécutive à l'indépendance. Ainsi Ivan Chichmanov écrit : "Nous sommes au seuil de deux cultures qui luttent entre elles pour gagner le grand prix. Il n'y a pas de raisons de croire que notre vieille culture (...) pourrait s'opposer éternellement à la nouvelle culture (...). Bientôt, chaque hameau éloigné ouvrira ses portes pour accueillir ces éléments nouveaux qui érodent toutes les particularités et tous les traits originaux des modes de vie populaire $" 9$.

Il va donc falloir mettre en œuvre des politiques culturelles et nationales pour préserver cette culture en danger de disparition. S'inspirant des mesures

\footnotetext{
${ }^{8}$ Dans son livre sur l'histoire de la Bulgarie, l'historien Ivan Iltchev caractérise cette époque comme une période de transition lors de laquelle s'accumulèrent les conditions et les moyens permettant aux Bulgares de revenir sur la scène historique : "L'économie du pays s'engage dans de nouvelles voies, depuis longtemps empruntées par les peuples de l'Ouest de l'Europe. En Bulgarie s'ouvrent les premières écoles dignes de ce nom. La mise en place d'un Exarchat autonome assure l'indépendance de l'Église sous la direction de prélats bulgares. La consolidation de l'organisation révolutionnaire permet de soulever la question bulgare devant les puissances européennes n. Itchev (Ivan). La rose des Balkans. Histoire de la Bulgarie des origines à nos jours, Sofia : Colibri, 2002, p. 111 ; pour plus des détails voir pp. 111-156.
}

9 Chichmanov (Ivan), " Znatchenieto i zadatchite na nachata etnografia " (L'importance et les objectifs de notre ethnographie), Sbornik za narodni umotvorenia, nauka $i$ knizhnina, 1, 1889, p. 5. 
prises ailleurs en Europe, le parlement édite en 1889 et en 1911 les premières lois consacrées aux collections et à la protection des vestiges et monuments historiques et culturels ${ }^{10}$. Ces textes réglementaient les conditions de création des institutions destinées à protéger le patrimoine national et à surveiller sa conservation. Vestiges et monuments représentaient explicitement le passé, ils devaient aider à rendre compte des origines et de la spécificité de la culture bulgare, en même temps que ces mesures contribuaient à développer une conception déjà normative du patrimoine, distinguant entre littérature, folklore, archéologie, ethnographie, architecture, art, etc. Parallèlement à cette politique de célébration de la culture nationale, comme partout en Europe les élites intellectuelles et les bourgeoisies urbaines vont créer des institutions muséographiques destinées à rendre compte de la richesse du folklore et de l'histoire locale. Ainsi, à Plovdiv, le musée archéologique est créé dès 1879 et le musée ethnographique en $1917^{11}$.

\section{PLOVDIV}

Plovdiv, seconde ville de Bulgarie, comprend à peu près 350000 habitants. Elle est située au sud du pays, dans la plaine de Thrace. Édifiée sur trois tépés (collines) au bord de la Maritza, elle fut, dès l'antiquité, une cité importante, sur la route entre la Grèce et les pays du centre et du nord de l'Europe. Sous Byzance, Trimontium, de son nom romain, est une ville florissante, jusqu'au peuplement par les Slaves au $\mathrm{VI}^{\mathrm{e}}$ siècle. Alors la ville reprend le nom thrace de Pulpudeva qui deviendra plus tard Plovdiv. Après la fondation de la Bulgarie danubienne (681), pendant plusieurs siècles Plovdiv passa alternativement des mains bulgares aux mains grecques, jusqu'à la conquête ottomane en 1364. La population bulgare diminua drastiquement au moment de la conquête ottomane et progressivement elle se stabilisa, au XVII ${ }^{\mathrm{e}}$ siècle, au sein des autres groupes qui habitaient la ville ${ }^{12}$. C'est ce que constatent plusieurs voyageurs, comme par exemple Adam Verner en 1616 : "Phillippopoli, nommé par les Turcs Filibé, est une ville assez grande, comparable à Neurdlingen (...). Il y a quelques belles églises, des minarets, un caravansérail, des bains. La ville est peuplée par des Bulgares, par des gens de Dubrovnik, des Grecs, des juifs qui

\footnotetext{
${ }^{10}$ Nedkov (Simeon), Musei i museologia (Musées et muséologie), Sofia : LIK, 1998, pp. 124 - 144.

${ }^{11}$ Ibid.

12 Gentchev (Nikolaï), Vazrojdenskiat Plovdiv (Plovdiv à l'époque de la Renaissance bulgare), Plovdiv : Chr. G. Danov, 1981, 17-41.
} 
s'occupent du petit commerce $"^{13}$. Sous le nom de Filibé, délaissant les collines en s'étendant dans la plaine à proximité de la Maritza, elle devint une cité orientale, centre d'une région riche et prospère : il y eut jusqu'à 55 mosquées, et sur la place du marché se côtoyaient plusieurs centaines de boutiques ${ }^{14}$.

A partir de la fin du XVIII ${ }^{\mathrm{e}}$ siècle, une bourgeoisie bulgare se constitue progressivement, faite de collecteurs d'impôts, de propriétaires de troupeaux de moutons qui exportent dans tout l'empire. Cette nouvelle élite s'installe à Plovdiv, investissant les vieilles collines délaissées par les Turcs. À côté de l'essor de l'artisanat et des débuts de l'industrie, une catégorie de gens lettrés apparaît qui se passionnent pour l'Occident, pour les idées de liberté, pour la langue française. Après la guerre russo-ottomane de 1877, le traité de San Stéfano impose aux Ottomans la création d'une principauté de Bulgarie dont Plovdiv est la ville la plus importante, peut-être aussi la plus cosmopolite. Vient alors ce que les Bulgares considèrent comme la trahison des grandes puissances, principalement la Grande Bretagne : le traité de San Stéfano est dénoncé, et la principauté est divisée en deux, une principauté de Bulgarie autour de Sofia, et la Roumélie orientale autour de Plovdiv, laissée sous le contrôle d'Istanbul. Rapidement, en 1885 , les deux entités seront réunies, mais entre temps Sofia est devenue la capitale politique du pays. Restera à Plovdiv d'être encore pour quelque temps sa capitale culturelle.

À cette époque la ville fait preuve d'une modernisation rapide comme en témoignent l'ouverture de la première école bulgare laïque, la première maison d'édition, l'essor de l'industrie. Ce sont des atouts qu'intellectuels et journalistes bulgares mettent en avant pour souligner la transformation accélérée de la ville et ses spécificités qui la rapprocheraient de villes européennes telles que Paris, Leipzig ou Manchester. Leur souci est alors, en insistant sur son caractère bulgare, de dépasser les questions liées à la co-existence des groupes ethniques nombreux. Réorganisation des espaces communautaires, changement de toponymie, glorification de la ville à travers la littérature contribuent à faire de Plovdiv un symbole du territoire national ${ }^{15}$. Pendant les guerres balkaniques qui vont suivre, entre 1912 et 1913, puis jusqu'en 1922, la Bulgarie est en conflit avec la Grèce et la Serbie, et Plovdiv va connaître de profonds bouleversements démographiques.

Comme souvent dans les vieilles villes d'Europe, chaque époque de l'histoire a laissé des traces. Du haut de l'une des collines de la ville, le panorama offre au regard toute la richesse de cette histoire : vestiges d'autels et de fortifications

\footnotetext{
${ }_{13}$ Tzvetkova (Bistra) (ed.), Tchujdi patepisi za Balkanite (Notes de voyages étrangères dans les Balkans), Sofia : Nauka i Izkusstvo, 1975, p. 143.
}

${ }^{14}$ Chichkov (Stoïo N.), Plodiv dans son passé et son présent, Plovdiv, 1926.

15 Kiossev (Alexander), Plovdiv : The Text of the City vs. The Text of Literature, Nexus Research Project 2000-2003. 
thraces ; agora grecque ; théâtre romain ; rempart médiéval ; bains turcs ; clochers des églises orthodoxes, catholiques et protestantes ; minarets des deux mosquées musulmanes ; église arménienne apostolique, synagogue, villas de la Renaissance bulgare, maisons art nouveau. Ce paysage urbain laisse apparaître ce qui est l'une des singularités de la ville : le nombre et la variété des communautés ethniques et religieuses qui l'ont habitée et qui l'habitent encore ${ }^{16}$.

À l'époque de la création des premiers musées, l'architecture civile n'est pas encore intégrée à la liste des éléments patrimoniaux qu'on cherche à sauvegarder. À la fin du $\mathrm{XIX}^{\mathrm{e}}$ siècle la municipalité engage l'architecte tchèque Josef Schniter pour préparer la modernisation de la ville. Il s'intéresse très peu à la vieille ville, et réalise le premier plan d'urbanisme en 1896 qui marque les limites du centre-ville. Celle-ci est formée des quartiers situés au pied des collines, largement construits par les Ottomans. Suivant les normes de l'urbanisme en vigueur dans l'ouest de l'Europe à l'époque, il détruit une bonne part du tissu urbain médiéval, dégage la place centrale et perce de grandes voies traversantes. Cette transformation importante ne concerne pas le territoire de la vieille ville bâtie sur les collines qui reste loin des préoccupations des aménageurs, certaines maisons tombent en ruines et cèdent la place à des friches où la nature va reprendre ses droits ${ }^{17}$.

Dans les premières décennies du $\mathrm{XX}^{\mathrm{e}}$ siècle, Plovdiv se développe rapidement et la croissance démographique est soutenue. En 30 ans la population double et la ville devient deux fois plus étendue. Il est donc nécessaire de l'adapter aux transformations profondes du contexte économique et social qu'elle connaît. Tout ce qui évoque son caractère cosmopolite, comme les nombreux bâtiments publics ottomans, est négligé, sinon largement détruit, l'optique des responsables municipaux étant la modernisation de la ville. En 1939

\footnotetext{
${ }^{16}$ Rezultati ot prebroiavaneto na Iztotchna Roumélia (Résultats du recensement en Roumélie Orientale), Plovdiv, 1885 ; Rezultati ot prebroiavaneto na naselenieto v Severna i Jujna Bulgaria (Résultats du recensement de la population de Bulgarie du Nord et du Sud), Sofia, 1888, 1892, 1901, 1905, 1910, 1927, 1934 ; Tzentralno statistitchesko upravlenie. Prebroiavane na naselenieto ot $1956 \mathrm{~g}$. (Administration centrale de sondages statistiques. Recensement de la population de 1956), Sofia, 1957; Prebroiavane na naselenieto na Bulgaria prez 1992. Izdatelstvo i petchat na Natzionalnia statistitcheski institut (Recensement de la population de Bulgarie en 1992. L'édition et l'imprimerie de l'Institut National statistique), Sofia, 1996 ; Prebroiavane na naselenieto na Bulgaria prez 2001. Izdatelstvo i petchat na Natzionalnia statistitcheski institut (Recensement de la population de Bulgarie en 2001. L'édition et l'imprimerie de l'Institut National statistique), Sofia, 2003 ; Koleva (Elena), " Promeni na etnitcheskia sastav na naselenieto na grad Plovdiv " (Changements de la structure de la population de la ville de Plovdiv), Godichnik na muzeite $\checkmark$ Plovdiv, $4,1965$.
}

${ }^{17}$ Topalova (Antoineta), Andreeva-Kertikova (Jordanka), « Hronologia, printzipi i priemsvenost pri gradoustroïstvenoto planirane na grad Plovdiv ot kraïa na XIX do 40-te godini na nachia vek n (Chronologie, principes et continuité dans la planification urbanistique de la ville de Plovdiv de la fin du XIX aux années 40 de notre siècle), Plovdiv po pâtja kâm modernija svjat, Plovdiv, 1998, p. 191 ; et entretien avec Antoineta Topalova. 
le Conseil municipal commande à Hermann Jansen, professeur à l'École Polytechnique de Dresde, un nouveau plan d'aménagement urbain. Celui-ci sera mis au point en 1944, développant une conception qu'on pourrait qualifier de pré-fonctionnaliste en distinguant les quartiers selon leurs fonctions. Même si à cause des changements politiques ce plan ne sera jamais appliqué, il est cependant intéressant de noter qu'il fonde déjà les transformations proposées sur l'idée " de sauvegarder les valeurs culturelles et esthétiques de la ville ${ }^{18}$. C'est la première fois que cette idée est exprimée dans un document officiel. Il prévoyait que la modernisation du tissu urbain et la création de nouvelles infrastructures devaient tenir compte de la sauvegarde de l'architecture de certains quartiers, en particulier celui construit sur les trois collines historiques, et de respecter le paysage particulier de Plovdiv.

Cette idée selon laquelle les trois collines représentaient un site historique et culturel qu'il fallait préserver parce qu'il constituait le principal héritage historique et culturel de la ville avait cours dans la bonne société plovdivienne depuis les années 20. Entre les deux guerres, les élites municipales se souciaient déjà de le préserver et s'inquiétaient de l'intégrer à la ville moderne. En 1937, le Conseil municipal vota un règlement d'aménagement du site qui prévoyait des dégrèvements d'impôts pour les propriétaires qui entretiendraient les vieux bâtiments. L'architecte Christo Péev ${ }^{19}$ s'associa au directeur du musée archéologique et à quelques personnalités locales afin de demander que des mesures soient prises pour sauvegarder le site des trois collines historiques et stopper la démolition programmée de plusieurs maisons ${ }^{20}$. Il parvint à mettre le maire de son côté et en 1942 la mairie définit juridiquement les limites de la vieille ville de Plovdiv, ce que le gouvernement confirmera par arrêté ministériel $^{21}$. On peut comprendre aujourd'hui, avec le recul, que, sans l'indiquer explicitement, ces décisions étaient prises dans le souci de souligner l'importance du patrimoine et son rôle dans les activités et l'aménagement de la ville.

\footnotetext{
${ }^{18}$ Ibid., p. 195.

19 Christo Péev (1893-1956), dont nous reparlerons plus loin.

${ }^{20}$ Entretien avec Véra Koralova qui fut architecte de la vieille ville et proche collaboratrice de Péev.

${ }^{21}$ Baltchev (Vladimir), " Obratite v kulturnia zhivot na Plovdiv i sadbata na Staria grad n (Les tournures de la vie culturelle de Plovdiv et le destin de l'Ancienne ville), Parvi tchetenija v pamet na Prof. T. Iv Jivkov, Plovdivski universitet, Plovdiv, 2002, pp.1-8.
} 


\section{LA MISE EN PLACE PROGRESSIVE D'UNE POLITIQUE PATRIMONIALE}

Après la deuxième guerre mondiale, le régime socialiste cessa pour dix ans toute politique de soutien au patrimoine architectural dans ses projets urbanistiques et la gestion du territoire, même si en 1951 est pris un arrêté prévoyant des financements publics pour la restauration des objets et monuments historiques $^{22}$. Dès le début des années 50 , le débat reprend sur la place que joue la vieille ville de Plovdiv dans le trésor national. Le pouvoir municipal demande alors un nouveau statut juridique et, en 1956, le ministère de l'Amélioration de la vie et de l'Architecture prend la décision, supervisée par le ministère de la Culture, suivant laquelle l'ancien quartier des trois collines est inscrit sur la liste des zones protégées au titre de la loi de sauvegarde des monuments de la culture $^{23}$. En même temps, la municipalité crée une cellule de gestion de l'Ancien Plovdiv qui, depuis la fin des années 6o, est abusivement appelée " Mairie " du Vieux Plovdiv. Son premier responsable est Atanas Krastev, l'homme clé du patrimoine et de la vie artistique de la ville pendant toutes les années 60 et 70.

Atanas Krastev, plus connu sous son surnom de " Natcho la culture " (Natcho Koultourata), né dans la ville voisine d'Assenovgrad, est arrivé à Plovdiv en janvier 1954, appelé par un de ses amis d'enfance pour devenir inspecteur au service "Science, art et culture " de la municipalité. Le jeune homme aimait déjà la peinture, mais il n'avait guère de goût pour les vieilles pierres. Dans ses attributions, on lui donne pourtant la responsabilité du secteur de la vieille ville. Elle est dans un état de vétusté avancé, un seul bâtiment a été restauré, en 1951, sous la direction de Christo Péev. Natcho se prend alors d'amour pour ce quartier qu'il ne quittera plus, s'installant dans l'une de ces maisons de la Renaissance bulgare, et il restera très marqué par sa rencontre avec l'architecte. À cette époque, la plupart des maisons du quartier ont été réquisitionnées par l'État pour en faire des logements sociaux. Ainsi, dans l'une des plus belles, la " maison de Hindlian ", édifiée en 1837 et qui possède de magnifiques fresques relatant les nombreux voyages de son propriétaire en Orient, ce sont jusqu'à 65 personnes qui seront logées dans une douzaine de pièces. C'est à ce même Péev que la municipalité et les services de l'État confient la tâche de faire une étude sur l'architecture de la période de la Renaissance bulgare dans cette partie de la ville. Cette grosse étude historique,

\footnotetext{
${ }^{22}$ Entretien avec Véra Kolarova : « En 1951 a été édicté le Décret du Conseil Ministériel n ${ }^{\circ} 1608$ consacré à la sauvegarde des monuments de la culture en Bulgarie. C'est le premier document officiel qui réglemente ces activités et, dès 1952, l'État commence à assurer régulièrement des financements de restauration n.

${ }^{23}$ Soit près d'une dizaine d'années avant la création des quartiers sauvegardés par André Malraux, en France.
} 
topographique, architecturale et sanitaire, sera la base à partir de laquelle seront réalisées les restaurations de plusieurs dizaines de bâtiments ${ }^{24}$.

En 1969, Atanas Krastev est nommé directeur de la toute nouvelle Division de gestion de la vieille ville qui prend la suite de l'ancienne " cellule " et rassemble trois personnes autour de lui. Une politique ambitieuse de restauration de la vieille ville est alors engagée, accompagnée par la mise en place d'un programme artistique et de valorisation qui permettra à la vieille ville de reprendre toute sa place dans vie locale. Il s'agissait de sauvegarder tous les monuments qui représentaient une étape ou une variante de l'architecture de la Renaissance bulgare, considérés d'un point de vue national autant que local. Chaque maison restaurée était ensuite affectée à un usage particulier. Il pensait - tout en suivant en cela une politique plus générale promue par l'État socialiste - que pour que ces restaurations puissent durer et se multiplier, les maisons devaient trouver une utilité sociale réelle ${ }^{25}$. Certaines furent transformées en musées, mais en général elles ont plutôt accueilli des organismes officiels ou associatifs : maison des écrivains (dans une villa où Lamartine séjourna quelques jours pendant son voyage d'Orient), maison des architectes ; ou bien elles étaient utilisées par des organismes publics pour accueillir des manifestations culturelles, des cérémonies protocolaires, ou pour loger les invités de marque d'un ministère ou de la municipalité : maison du ministère des Affaires Étrangères, maison du ministère des Relations Économiques, etc. Le but de Natcho était d'attirer dans la vieille ville tous les Plovdiviens qui étaient actifs dans le domaine de l'art et de la culture, toutes les personnes de marque de passage, de faire connaître la vieille ville dans toute la Bulgarie et au-delà. Un autre versant de la politique culturelle mise en place fut de développer salons de peinture, manifestations culturelles et fêtes populaires. Nous y reviendrons.

L'une de ces maisons, la maison dite Balabanov, fut entièrement reconstruite en 1978 d'après les plans que Péev avait recueillis, afin d'en faire le " petit palais de Plovdiv ". Cette construction entièrement neuve, mais édifiée selon les techniques traditionnelles, est la réplique la plus exacte possible d'une bâtisse du début du XIX ${ }^{\mathrm{e}}$ siècle, démolie dans les années d'après guerre, sur l'emplacement de laquelle une maison moderne avait été bâtie en 1958. Son histoire est édifiante des enjeux et des ambiguités de ces opérations. La qualité de la construction, la justesse du goût des décorations intérieures ne permettent pas à un profane de repérer le pastiche ${ }^{26}$. Encore aujourd'hui, dans les circuits

\footnotetext{
24 Elle fera l'objet d'un ouvrage intitulé Les maisons de la ville de Plovdiv pendant l'époque de la Renaissance bulgare, publié en 1960 sous la direction de l'architecte Emile Momirov.

${ }^{25}$ Entretien avec Atanas Krastev : « Notre objectif général visait à sauvegarder ce territoire comme un organisme vivant de la ville où les gens continueraient à habiter $n$.

${ }^{26}$ Certes, les câbles électriques et tuyauteries sont invisibles, insérés dans les murs...
} 
de visite qui sont organisés, dans les plaquettes touristiques, cette particularité n'est jamais évoquée ${ }^{27}$, signe parmi d'autres de l'ambiguïté qui continue de prévaloir sur l'usage emblématique de ces lieux.

Pourquoi construire une maison neuve alors qu'il en existe des dizaines alentours ? Répondait-elle seulement à un réel besoin de créer un lieu de spectacle et de culture dans la vieille ville ? S'agissait-il d'un " geste architectural " guidé par le goût du panache ? D'une expérimentation destinée à mieux préparer les restaurations suivantes ? Nous n'avons pas la réponse précise. Peut-être s'agissait-il simplement de l'ambition personnelle de Atanas Krastev, au moment de sa plus grande gloire, de faire renaître celle qui était considérée comme la plus belle maison du site. Il fit reconstruire de la même façon une maison de XVIII ${ }^{e}$ siècle qui sert encore aujourd'hui à héberger l'administration de la vieille ville. Ce désir, qui peut paraître inconsidéré, de montrer une image de Plovdiv la plus fidèle possible aux représentations qu'on en avait de l'époque de la Renaissance bulgare, n'est finalement qu'un exemple parmi d'autres, peut-être l'un des plus emblématiques, d'une politique plus globale visant à valoriser l'idée nationale après l'internationalisme qui avait prévalu pendant la première décennie du régime communiste. Un autre fait mérite d'être souligné : Balabanov était un riche marchand bulgare, peut-être l'un des plus riches de la ville au milieu du $\mathrm{XIX}^{\mathrm{e}}$ siècle, mais il acheta cette maison à un autre marchand, qui semble avoir été un commerçant grec, Hadji Lampcha ${ }^{28}$ : nous voyons ainsi comment l'idée nationale s'est construite, audelà des différents régimes politiques, en " bulgarisant " des héritages au caractère parfois multiculturel.

Dans son ouvrage posthume, Péev cherche à identifier les influences esthétiques de la maison de Plovdiv et à en dresser une typologie. Il distingue trois groupes de maisons. Le premier est constitué des bâtisses les plus anciennes, datant du XVIII ${ }^{\mathrm{e}}$ siècle, à l'architecture sobre correspondant au style de vie simple d'une population d'artisans et de petits commerçants, proches des maisons " ouvertes " des régions rurales alentours. Le deuxième groupe est constitué de maisons plus récentes, plus vastes, aux formes et aux détails architectoniques et décoratifs d'une grande variété, munies souvent d'étages en encorbellement. Elles sont entourées de petits jardins très soignés, intimement associés à la vie de la demeure, munis de fontaines, de patios, de grands arbres,

\footnotetext{
${ }^{27}$ Le dépliant touristique qui montre les cinq plus beaux édifices de la vieille ville indique à son sujet (en anglais) : " A symetric building, built at the beginning of the $19^{\text {th }}$ century. The first floor is occupied by contemporary painting exhibition. On the second floor, used in the past by its owner for a guest room, you could see an urban Renaissance style interior (...) $n$.

${ }^{28}$ Entretien avec Atanas Krastev : "Les temps étaient comme ça, il fallait donner au nom de la maison une sonorité plus bulgare, et c'est pourquoi on l'avait appelée la maison Balabanov (...), mais, en fait, c'est la maison de Hadji Lampcha ". Le surnom de " Hadji " était donné, en signe de respect, aux chrétiens qui avait fait le pèlerinage de Jérusalem.
} 
de rocaille. L'influence de l'architecture urbaine ottomane y est forte, celle-ci ayant été elle-même influencée par le baroque italien qui a pénétré dans l'empire au XVII ${ }^{\mathrm{e}}$ siècle à partir de Constantinople. Les plafonds, en bois, sont particulièrement soignés et décorés, les murs sont recouverts de fresques qui peuvent rappeler les activités du propriétaire, l'intérieur est souvent organisé autour du salon, de forme rectangulaire ou ellipsoïdale, pièce stratégique pour ces marchands très attentifs au décorum et à l'ostentation de leurs intérieurs. Ces maisons présentent toutes une symétrie rigoureuse de leurs plans intérieurs et de leurs façades qui rappellent les architectures urbaines populaires de la région. Un troisième groupe rassemble des maisons asymétriques. À partir du milieu du XIX ${ }^{e}$ siècle, des spécificités architectoniques nouvelles apparaissent avec l'utilisation généralisée du pan de bois et de la brique, des corniches en lattis crépis de plâtre, la disparition des encorbellements.

À partir de ces éléments, Péev définit un style architectural qu'il nomme " baroque oriental ". Cela sera l'objet de débats passionnés, tant au sein de l'Académie bulgare que dans la presse, autour de l'hypothèse que ces maisons, certes " bourgeoises ", pouvaient relever ou non d'une architecture populaire, ou s'il fallait considérer qu'elles étaient surtout inspirées de modèles extérieurs, ottomans ou baroques. Ainsi une partie des architectes s'est élevée contre cette notion de " baroque oriental " au motif qu'elle gommait les capacités créatrices propres de la population locale ${ }^{29}$. Quelques dix années après la parution de l'ouvrage, l'architecte Georgi Kojuharov, de l'Institut d'urbanisme, écrit dans le journal hebdomadaire Culture populaire :

Le modèle de la grande maison plovdivienne va se diffuser au sein de la bourgeoisie dans toutes les régions de Bulgarie. Les maisons plovdiviennes forment ainsi le type d'architecture qui exprime les meilleures œuvres de la créativité populaire en matière d'architecture pendant l'époque de Renaissance bulgare. (...) Elles représentent le degré le plus haut du développement historique de la maison populaire ${ }^{30}$.

Ainsi, selon ce spécialiste, la maison plovdivienne est un exemple de développement autonome de l'architecture populaire, elle en est même le modèle le plus abouti qui va se répandre sur tout le territoire bulgare. Cette opinion, fidèle à l'idéologie socialiste qui privilègie le rôle des " masses populaires " dans les changements historiques, met en avant la capacité créatrice des populations qui seraient à l'origine du caractère proprement bulgare de ce style architectural en minorant les apports stylistiques extérieurs. In fine elle permet de renforcer l'idéologie patrimoniale et populiste que la politique patrimoniale a surtout pour vocation d'illustrer.

\footnotetext{
29 Entretien avec Antoineta Topalova.
}

$3^{\circ}$ Kojuharov (Georgi), "Plovdivskata vazrozhdenska kachta " (La maison plovdivienne de l'époque de Renaissance), Narodna cultura, (23), 5 mai 1971. 


\section{LES ENJEUX IDÉOLOGIOUES DE LA POLITIDUE DU PATRIMOINE}

Ces débats savants sur la typologie architecturale peuvent être mis en parallèle avec les difficultés qu'il y eut pour étendre les mesures de protection au quartier de Kapana, le quartier artisanal de l'époque ottomane bâti à partir du $\mathrm{XV}^{\mathrm{e}}$ siècle au pied de trois " tépés " (les collines), derrière la grande mosquée Djumaja Djamia. Dans les années 70, alors qu'un concours d'architectes de 1968 avait prévu de le détruire entièrement pour y installer un vaste centre commercial, des architectes comme Antoineta Topalova, qui était alors l'architecte en chef de la Ville, s'inquiètent de la protection du quartier. Les travaux de démolition furent partiellement stoppés mais il faudra attendre 1989 pour que la mesure de protection soit effective.

Ces deux exemples peuvent être interprétés comme des expressions des enjeux politiques et idéologiques qui entourent alors les processus de patrimonialisation. Certes, il y a des questions financières : investir largement sur les trois collines se fait au détriment d'autres investissements dans la ville, et en particulier au détriment de la restauration des quartiers ottomans, vestiges d'autres périodes de l'histoire. On peut aussi penser, en écoutant les propos d'Antoineta Topalova, qu'il y avait derrière ces débats des questions de doctrine très proches de celles qui étaient en vigueur en France ${ }^{31}$ à la même époque sur la place à donner à l'architecture civile et populaire dans la protection du patrimoine ${ }^{32}$. Cependant, limiter la protection à des demeures luxueuses héritées de la période qui précède l'indépendance, des demeures pour l'essentiel bâties par des Bulgares, même s'il y eut parmi eux des marchands juifs, arméniens ou grecs ; ou bien l'étendre à des quartiers plus populaires, édifiés par " l'occupant ottoman " selon un urbanisme très " oriental ", dépasse la simple question de doctrine architecturale et patrimoniale. Ce sont des représentations collectives de l'histoire qui sont alors pensées, produites et appliquées, des représentations qui vont concerner bien d'autres sphères de la vie publique. Tous les régimes politiques ont eu à cœur, particulièrement depuis le XIX ${ }^{\mathrm{e}}$ siècle ${ }^{33}$, d'asseoir leur légitimité sur l'histoire. Dans les pays d'Europe centrale, les indépendances se sont accompagnées de la valorisation des cultures

\footnotetext{
${ }^{31}$ Il semble d'ailleurs que des architectes bulgares venaient assez régulièrement en France, qu'ils suivaient de près les réflexions et les expériences de leurs collègues français de l'Atelier d'urbanisme et d'architecture. (Entretien avec A Topalova). Antoineta Topalova elle-même fit des séjours en France.

${ }^{32}$ Sur le sujet, voir Poirrier (Philippe), Vadelorge (Loic), Pour une histoire des politiques du patrimoine, Paris : Comité d'histoire du ministère de la Culture / Fondation Maison des sciences de l'homme, 2003.

33 Voir les travaux de Thiesse (Anne-Marie), La création des identités nationales. Europe XVIII-XX ${ }^{e}$ siècles, Paris : Seuil, 1999.
} 
populaires, suivant le modèle germanique inspiré des thèses de Herder ${ }^{34}$. Le régime communiste, dans sa politique patrimoniale, s'inscrit ici dans la continuité de ses prédécesseurs ; seul l'objet patrimonialisé change : ce ne sont plus les grands poèmes épiques d'Ivan Vazov35, les musées archéologiques ou ethnographiques, les chapelles et les monastères, ce sont les productions architecturales civiles qu'il va falloir faire entrer dans la production culturelle nationale. Le problème qui se pose alors aux spécialistes de l'époque, savants, architectes, ou fonctionnaires de l'administration culturelle, est de savoir comment traiter cette architecture, inspirée de l'extérieur et bourgeoise, dans un régime communiste qui, à partir du milieu des années 50 , va défendre une conception très nationale et populaire de la culture. Deux questions se posent alors. La première est de comprendre comment s'articulent protection du patrimoine et discours sur le patrimoine (et singulièrement, pour le cas qui nous intéresse ici, ce qu'on appelle la " valorisation du patrimoine ") ; la seconde est qu'il faut replacer l'analyse à un niveau national, et pas seulement à celui de la ville de Plovdiv. Or ces deux questions sont intimement liées.

Les prérogatives de la division de la gestion de la vieille ville incluaient, outre la conservation des bâtiments anciens, l'aménagement du lieu et la création de nouvelles infrastructures qui devaient respecter le caractère patrimonial des trois collines. Des tranchées étaient ouvertes dans les espaces verts afin de permettre de lire la stratigraphie complexe du site de la ville. Il s'agissait, à travers ce dispositif, de donner à voir la " bulgarité " des vestiges archéologiques et des monuments dans toute leur profondeur historique, depuis l'époque thrace jusqu'à l'indépendance. On parle alors de "site - musée ", de " partie muséale de la ville ". Selon Mme Vera Kolarova, architecte à l'Institut régional des monuments de la culture qui fut longtemps détachée auprès d'Atanas Krastev pour prendre en charge la supervision des travaux de restauration de la vieille ville, il fallait donner " la priorité à la sauvegarde dans leur intégrité à des monuments de l'époque de la Renaissance bulgare [parce que] l'Ancien Plovdiv est un ensemble architectural vital de [cette] époque ".

En fait, cette politique locale était très encadrée par les structures de l'État et par le parti communiste. En témoigne les réserves qui accompagnèrent la promotion d'Atanas Krastev à la tête de la division de la gestion de la vieille ville, car on le jugeait peu apte à faire valoir les intérêts de l'opération auprès du comité central du parti. C'est, selon lui, grâce aux relations personnelles 
qu'il avait su établir avec des artistes de tous horizons qu'il obtint finalement gain de cause ${ }^{36}$. La politique patrimoniale appliquée à Plovdiv s'inscrit dans le cadre d'un vaste plan de valorisation du folklore et des identités nationales engagé à partir des années 60 par le Parti communiste bulgare sous la direction de la responsable du Comité de la culture et des arts, Ludmila Jivkova, fille de Todor Jivkov, le premier secrétaire du Parti. Il s'agissait, en partant du patrimoine, de reconstruire les cultures locales pour les insérer dans une idéologie plus large de valorisation des cultures populaires. Sont alors protégés des villages entiers transformés en musées de site (par exemple ceux de Jeravna, Bojentzi ou de Koprivchtitza), des villes ou quartiers urbains (par exemple la ville ancienne de Tarnovo, capitale historique du royaume bulgare), des édifices comme le monastère de Batchkovo fondé au XII ${ }^{\mathrm{e}}$ siècle par les frères Bakouriani, des nobles géorgiens, ou des édifices plus isolés comme par exemple, à Plovdiv, le bâtiment des bains qui date du milieu du XVI ${ }^{\mathrm{e}}$ siècle. Les expertises permettant la sélection des édifices à sauvegarder étaient produites par l'Institut national des monuments de la culture, créé en 1957, qui les classait selon leur caractère mondial ${ }^{37}$, national, régional ou local ; à partir des années 80 il propose d'étendre éventuellement la protection au site environnant l'édifice. En 1965 est créé à Plovdiv un Institut régional des monuments de la culture dont les architectes et techniciens vont être mis au service de la restauration des bâtiments, sous la tutelle des chefs de projets, comme l'était Atanas Krastev. Les frais de fonctionnement sont assurés par la municipalité, et les investissements par l'État.

Dans les années 70, on revisita également les origines de la nation bulgare en multipliant les chantiers de fouilles archéologiques permettant de faire valoir les liens de continuité depuis l'époque thrace. Un vaste programme de festivité fut programmé pour célébrer les " 1300 ans de l'existence de la Bulgarie " mettant en relief une vision de l'identité nationale appuyée sur l'osmose des trois ethnies : Thraces, Slaves, et proto-Bulgares. Dans le même temps, quelques artistes connus tels quels Tzanko Lavrenov ou Zlatju Bojadjiev peignaient des tableaux montrant Plovdiv à l'époque de la Renaissance bulgare. En mettant ces maisons en image, ils contribuèrent à formaliser un style architectural national, ils donnèrent forme à l'imaginaire national ${ }^{38}$.

\footnotetext{
${ }^{36}$ Dans le Livre d'or qu'il a conservé, on peut lire les noms - associés à des annotations chaleureuses ou à des croquis - de Alexis Weissenberg, Yordan Raditchkoff, Tsvetan Todorov, Gabriel Garcia Marquez, Picasso, ...

37 Dès 1979, quatre lieux et édifices sont classés par l'Unesco au titre du patrimoine culturel mondial, dont la tombe thrace de Kazanlak.
}

${ }^{38}$ Ditchev (Ivaylo), Ot prinadlezhnost kam identitchnost (De l'appartenance à l'identité), Sofia : LIK / DW, 2002, p. 117. 


\section{LA FÊTE DE L'ANCIEN PLOVDIV ET LES SALONS DE PEINTURE}

Apres la découverte archéologique du théâtre romain consécutive au percement d'un tunnel routier sous la vieille ville, Natcho convainc les autorités de la nécessité de mettre à jour le site antique et de détruire les immeubles édifiés sur son emplacement. Cela prendra de longues années. Le déblaiement sera effectué par une brigade de volontaires, pour la plupart des étudiants de l'École des Beaux Arts de Plovdiv, entre 1979 et 1981. Mais, avant même la valorisation du site, cette découverte va singulièrement dynamiser les programmes culturels et patrimoniaux dans la ville. En 1969 a été créé un comité national de l'Ancien Plovdiv associant représentants de l'État, du parti et de la ville39. L'objet de cette structure interministérielle était d'élaborer une stratégie de développement pour l'Ancien Plovdiv, y compris l'animation culturelle ${ }^{40}$. Dès 1970, ce comité propose un programme ambitieux dont l'objectif est de valoriser culturellement les modes de vie des époques passées pour en faire une matrice de la culture contemporaine et favoriser le tourisme. Il s'agissait plus clairement d'encadrer les formes collectives d'appropriation d'un patrimoine dont l'intérêt politique et idéologique était d'exprimer une synthèse entre l'histoire locale et le discours sur l'identité nationale.

Ce programme proposait également le développement du tourisme sur le site. Pour Mme Elena Ouzounska, qui était conservatrice de la vieille ville, l'objectif était de transformer le territoire de l'Ancien Plovdiv en " Montmartre bulgare ". Cette idée est aujourd'hui largement partagée par les gens de culture de Plovdiv ${ }^{41}$. S'inspirant peut-être de la première exposition artistique de plein air qui avait été organisée par Natcho le $1^{\mathrm{er}}$ avril 1969, à l'occasion de la fête qui eu

39 Il était composé de représentants du ministère du Commerce, du Comité national au tourisme, du Comité national à la culture et à l'art, du Comité national de la jeunesse et du sport, de l'Institut national des monuments culturels, de la "Mairie de l'Ancien Plovdiv n, du Bureau politique du Parti communiste.

$4^{\circ}$ On trouve cette information dans les journaux quotidiens : Matheev (Matheï), « Drevnata tchast na Plovdiv, edin vazobnoven arhitekturen komplex " (L'ancienne partie de Plovdiv, un ensemble architectural renaît), Vetcherni novini, (6048), 11 mars 1971 ; Rédaction, " Za uskorenoto izgrajdane na dreven Plovdiv n (De la construction accélérée de l'ancien Plovdiv), Otetchestven glas, (8186), $1^{\mathrm{er}}$ avril $1971 ; \mathbf{M e l e v}$ (Georgi), " Mladostta na dreven Plovdiv (perspektivna programa) " (La jeunesse de l'ancien Plovdiv [programme prospectif]), Otetchestven glas, (8196), 13 avril 1971.

41 " On peut dire que l'Ancien Plovdiv est considéré comme une réplique artistique de Montmartre. Cette idée donnait le moral aux artistes peintres de Plovdiv puisqu'ils pouvaient montrer des similitudes avec Paris tandis qu'à Sofia cela n'était pas possible. (...) Parce que dans ce système politique fermé oủ nous vivions dans les années 50-60, l'information n'arrivait pas facilement (...). La seule manière que nous avions de montrer que nous prenions part au monde, c'était de faire les choses comme elles se faisaient ailleurs. C'était aussi une attitude d'indépendance d'esprit que de créer la même ambiance à Plovdiv qu'à Paris, c'est parce que nous avions toujours une richesse culturelle qui compensait ... " (entretien avec Krassimir Linkov). 
lieu pour célébrer la création de la " mairie " de l'ancien Plovdiv, il recommandait l'organisation d'activités culturelles (expositions, concerts, lectures publiques) en liaison avec la Foire internationale qui se tient à la fin du mois de septembre.

Cette manifestation du $1^{\text {er }}$ avril va avoir une importance considérable dans l'histoire culturelle de la vieille ville. Elle marque l'institutionnalisation de la " mairie " du vieux Plovdiv, les débuts d'une ère nouvelle pour la peinture qui va bénéficier d'une véritable scène locale et nationale, et elle est souvent considérée comme l'acte fondateur de la fête du vieux Plovdiv qui connaitra quelques années plus tard un franc succès. Revenons sur l'exposition. Natcho avait invité plusieurs peintres de la ville, et en particulier deux jeunes artistes qui se tenaient à l'écart du réalisme socialiste, chefs de fille de ce qu'on nomme alors " l'école de Plovdiv ", Georgi Bojilov-Slona (1935-2001) et Dimitar Kirov (1935). Elle se tenait sur une petite place à proximité d'un des principaux monuments du quartier, l'église Saints Konstantin et Elena, et tous les passants, touristes ou habitants du quartier pouvaient s'y arrêter. Bien qu'organisée sans l'accord du Parti communiste, il n'y eut pas de réactions négatives de la part des autorités. L'année suivante il invita de nombreuses personnalités politiques, y compris Todor Jivkov, le premier secrétaire du Parti communiste, pour leur montrer la vieille ville et leur expliquer son action. Entre attention aux politiques nationales et un goût certain pour l'indépendance d'esprit, Natcho va mettre une grande énergie à appliquer ce programme. Pour ce faire, les maisons d'une ruelle sont transformées en ateliers artisanaux, certaines villas de la Renaissance bulgare deviennent des musées ou bien sont utilisées pour des réceptions ou pour accueillir les hôtes de marque, comme nous l'avons déjà vu. Pour favoriser l'animation du site il organise fêtes, festivals et salons divers dont certains traverseront les bouleversements politiques.

Même si elle ne s'installe durablement dans le paysage des activités culturelles qu'en 1979, la fête du Vieux Plovdiv, qui va reprendre bien des éléments de celle de 1969 et dont on considère généralement qu'elle en est la continuation, est l'une des manifestations qui illustre le mieux la manière dont est traduite cette politique publique. La manifestation est pédagogique, encadrée par les représentants de la ville et du Parti communiste, mais elle laisse également entrevoir un jeu d'acteurs qui est souvent affiché, dans les propos tenus au cours de nos entretiens, comme une singularité locale : un savoir faire bien rodé, parfois qualifié de " méridional ", associé à un certain art de vivre plovdivien. Celui ci permettait d'adhérer à un discours idéologique de la continuité de la culture bulgare à travers les différents régimes politiques tout en maintenant un certain degré d'improvisation des habitants qui affichent, dans les marges de l'action culturelle, leur goût de la liberté. Organisée chaque dernier dimanche du mois de septembre, cette fête rassemble concerts, spectacles, expositions, lectures etc. autour d'un événement central qui est un défilé carnavalesque. 
Lors de la première fête, un artiste avait fabriqué les mannequins caricaturant des personnes connues de Plovdiv - le maire, les artistes, Natcho. Ces mannequins étaient au centre de la fête. [On partait] du centre de la ville, dans la rue principale, et on arrivait devant le bâtiment de la " mairie " de l'Ancien Plovdiv. Ici sur une grande scène se relayaient les spectacles, concerts, etc. (...) Nous inventions les petits détails pour reconstituer l'ambiance de l'époque de la Renaissance (...) Une année, le musée ethnographique nous a prêté les costumes et il y avait des gens qui se sont promené toute la journée en portant ces habits... $4^{2}$

L'objectif affiché était d'attirer l'attention des habitants et des visiteurs sur le patrimoine architectural en reconstituant l'ambiance d'une époque considérée comme glorieuse, pendant laquelle la nation bulgare moderne avait pris conscience de son unité politique et culturelle. Le spectacle était construit sur les caractéristiques de la fête urbaine ancienne, telle qu'on peut les imaginer en voyant certaines toiles rassemblées au musée de peinture, particulièrement celles de Zlatiu Boiadjiev ou de Kosta Forev. Aujourd'hui encore cette fête, qui passe dorénavant pour " traditionnelle ", est marquée par la forte participation des habitants de Plovdiv, en particulier ceux de la vieille ville qui continuent à être très actifs dans sa préparation.

Autres manifestations devenues emblématiques de la vie culturelle et artistique de la vieille ville, les salons de peinture. En 1967, Natcho avait organisé dans sa propre maison une première petite exposition d'aquarelles de Georgi Bojilov, invitant surtout des amis. En 1969 comme nous l'avons vu, mais aussi les années suivantes, il renouvela de manière sporadique ces événements en exposant des tableaux d'artistes différents dans les cours des maisons restaurées de la vieille ville. Les journaux et les revues spécialisées insistèrent d'emblée sur la force du lien qui unissait la peinture et le cadre patrimonial de la ville : "L'architecture et les couleurs de l'Ancien Plovdiv, comme celles de Koprivchtitsa, de Karlovo ou de n'importe quelle ancienne ville bulgare, permettent de bien mettre en relief le caractère national de notre art contemporain "43. C'est le début du Salon d'automne dont l'objectif était de présenter les meilleures oeuvres des artistes bulgares, de confirmer la particularité de Plovdiv comme centre artistique et d'éprouver le niveau de l'école de peinture de la ville.

Un règlement permettait de régler la participation des artistes au salon d'automne. La direction de la culture de la ville organisait un jury avec des créateurs célèbres, des critiques d'art, des représentants de l'association des peintres plovdiviens, des représentants de la municipalité et du comité de

43 Tzokova (Eva), "Izlozhbi, koito postaviat problemi " (Expositions qui posent des problèmes), Izkusstvo, (9-19), 1970, pp. 55-57. 
l'Ancien Plovdiv. Sur la base de la présentation de deux ou trois tableaux et de leurs curriculum vitae, le jury invitait huit ou neuf personnes parmi la centaine de peintres qui répondaient à l'appel à exposition. Pour beaucoup de gens que nous avons interviewés, ce mode d'organisation permit longtemps le prestige du salon. Avec la grave crise économique du début des années 9o, le système mis en place ne fonctionna plus. En 1996 un autre mode de fonctionnement fut mis en place. Le salon continua à être financé par la municipalité, mais elle en confia l'organisation à un commissaire d'exposition unique qui, autour d'une thématique et sur la base d'un projet défendu devant les responsables locaux, choisissait lui-même les artistes invités 44 . À chaque fois, un artiste connu de Plovdiv était choisi comme invité d'honneur.

Une autre manifestation commence en 1985 , le Salon de plein air qui aura lieu chaque année à la fin de l'été. Il a deux responsables, le peintre Mateï Mateev, et une représentante de la municipalité, Tania Sardanova. La municipalité de Plovdiv invite dix artistes de pays européens différents qui restent ensemble pendant dix jours dans la maison des peintres dans la vieille ville. Ils sont invités à voyager dans la région, à faire connaissance avec la culture bulgare. À la fin de leur séjour, ils présentent leurs œuvres au public et en laissent deux pour la ville, déposées à la " Mairie " de l'Ancien Plodiv qui se constitue ainsi un fond de toiles contemporaines. À travers cette manifestation, il s'agit de favoriser les rencontres d'artistes, mais aussi de présenter l'art bulgare et plovdivien à des artistes étrangers.

\section{LA PEINTURE À PLOVDIV : UNE HISTOIRE DÉJÀ ANCIENNE.}

Ces liens étroits entre Plovdiv et les peintres a une histoire déjà longue qui débute peu de temps après l'indépendance du pays. En 1911, au moment de la foire internationale qui se tient alors dans la ville, un groupe de peintres plovdiviens avait décidé de faire valoir son autonomie par rapport à Sofia en organisant la première exposition internationale de peinture du pays 45 . Tenaillés entre les influences étrangères (principalement l'Expressionnisme allemand, le Symbolisme mais aussi l'Impressionnisme ou, plus tardivement, le Cubisme) et le tropisme national, ces peintres voyageaient beaucoup mais revenaient le plus souvent au pays. En 1919, apparaît un mouvement esthétique intitulé " Rodno izkoustvo" (l'Art natal) qui réunissait des artistes, des intellectuels, des

44 La première fut Galina Lardeva et l'invité d'honneur fut Georgi Bojilov-Slona. En 2002 le commissaire était Dimitar Grozdanov, artiste phare du courant de " l'Art non conventionnel ".

45 La thèse de doctorat de Krassimir Linkov, dans laquelle il a développé ce sujet, est consacrée à l'histoire de la vie artistique des peintres de Bulgarie du sud. Ici nous nous appuyons aussi sur son entretien. 
philosophes. Le mot d'ordre était de bâtir les fondements d'un art moderne, en même temps européen et proprement bulgare, qui se baserait sur " l'esprit national ". L'un de ses principaux représentants, le poète, philosophe et artiste Guéo Milev, écrit : " L'homme devient un habitant de la planète Mars s'il n'est pas habité par le sentiment national. Le sentiment national est une force spirituelle qui conduit les activités créatrices parce qu'il repose dans le subconscient ". La critique d'art Rouja Marinska note que pour les adhérents du mouvement les qualités artistiques des œuvres étaient liées au caractère national de l'art. Elle a constaté que la plupart de ces artistes privilégiaient les thèmes appartenant au folklore et à l'histoire afin de symboliser l'esprit bulgare. On trouve dans leurs œuvres de nombreuses représentations de paysages emblématiques de la nature et de l'histoire bulgare : monastères et églises orthodoxes sont des sujets de prédilection, ainsi que des scènes recomposées de la vie " traditionnelle " des paysans ${ }^{46}$. Malgré l'importance démographique qu'ils continuent à représenter, Turcs et Bulgares islamisés 47 sont quasiment absents de ces peintures, car on voulait attirer l'attention surtout sur les temps glorieux de l'histoire médiévale. Sur le plan formel, ces peintres reprirent les techniques picturales et les types d'ornementation du Moyen âge bulgare. L'objectif était de créer, à travers l'image, un lien imaginaire entre " les siècles d'or de la culture bulgare " et l'époque contemporaine.

Dans les années 50, l'art monumental, influencé par le muralisme mexicain, connaît un réel succès en Bulgarie. Mais la formation académique des artistes est médiocre, les voyages hors du monde soviétique très difficiles ; alors les artistes apprennent les uns des autres, au cours d'échanges informels et de rencontres. Autour des anciens maitres d'avant guerre, se développe à Plovdiv un véritable milieu de peintres et de poètes qui se retrouvent régulièrement dans des cafés de la vielle ville ou à l'école des beaux arts. Quelques grandes figures émergent, plutôt iconoclastes et peu respectueuses des canons de l'art officiel, dans un contexte national peu porté à la fantaisie : Zlatiu Boiadjiev (1903-1976), auquel la ville consacrera un musée, et Tzanko Lavrenov (1896-1978) pour la première génération; Georgi Bojilov-Slona, Dimiter Kirov et quelques autres pour la suivante. Les premiers avaient commencé la peinture avant la Seconde Guerre mondiale, et ils continuèrent sous

\footnotetext{
${ }^{46}$ Marinska (Rouja), 20-te godini v balgarskoto izobrazitelno izkustvo (Les années 20 des beaux arts bulgares), Sofia : Otvoreno obchtestvo, 1996.

47 Ils portent le nom de Pomaks. La colonisation des Balkans par les Ottomans s'est accompagnée de conversions à l'islam, choisies ou forcées, d'une partie des populations autochtones. En Bulgarie, on considère en général deux régions où il y eut une islamisation massive : les montagnes des Rhodopes, proches de Plovdiv, et la région de Lovetch. L'État bulgare eut une politique de conversion au christianisme qui eut quelques résultats jusqu'en 1989. Aujourd'hui, ces personnes se répartissent selon trois positions quand on les interroge sur leurs origines : certains revendiquent une origine bulgare, d'autres une origine turque, et les derniers déclarent être les descendants de la population souche du pays, les héritiers des tribus thraces.
} 
le régime communiste sans qu'il soit très aisé de distinguer dans leurs œuvres entre les deux périodes. En 1969 on pouvait lire dans une revue spécialisée :

Nous appelons Plovdiv " la ville des peintres " pas seulement à cause de son riche passé, mais surtout à cause de son présent. Le groupe des artistes de Plovdiv est un groupe très actif qui rassemble des peintres qui ne vivent pas dans la capitale. Les styles différents et l'individualité des artistes sont les traits de base de "l'école plovdivienne ". Ses marques distinctives sont : des sujets contemporains, la richesse de la palette, le sentiment d'appartenance au pays natal ${ }^{4}$.

Loin de Sofia, d'une génération à l'autre, un certain vent de liberté souffle chez ces artistes dont certains vont développer un art à la fois personnel et marqué par un attachement singulier à la ville et sa région. Aujourd'hui ces artistes sont sensibles aux questions environnementales, ils s'interrogent sur les problèmes de l'existence, de la solitude, sur l'individualisme montant dans la société. Comme beaucoup d'artistes bulgares, ils ont aussi une prédilection pour la nature, la montagne, un certain mysticisme qui explosera après 1989 . Jusqu'à une période récente, des artistes tels que Kosta Forev (1942-1993) ou Mateï Mateev ( 1945 -) vont exprimer avec force leur attachement pour la vieille ville, prenant les anciennes demeures ou les rues comme sujets, privilégiant le cadre du site des trois collines, exprimant la vitalité des fêtes urbaines, peignant de manière réaliste ou burlesque des scènes de la vie quotidienne dans lesquelles il n'est pas rare de retrouver des personnages publics locaux plus ou moins caricaturés. A posteriori, les critiques ont plutôt insisté sur la dimension non officielle de cet art qu'on a pu opposer au réalisme socialiste, alors même que les jurys des concours des salons qui les ont fait connaître étaient des plus officiels.

\section{CONCLUSION : PATRIMOINE, ARTISTES, ET ANIMATIONS CULTURELLES POUR FABRIQUER UNE VILLE IMAGINEE}

Certes, la politique patrimoniale et le développement artistique qu'a connus Plovdiv étaient largement encadrés par les choix réalisés en haut lieu, au niveau de l'État et du Parti. On ne peut pas nier toutefois qu'ils doivent beaucoup à l'énergie et à l'engagement de quelques individus, au premier rang desquels il faut évoquer l'architecte Péev et le premier responsable de l'administration culturelle de la vieille ville, " Natcho la culture ". Cependant, le fait que cette notoriété ait des racines plus lointaines, inscrites au cœur de l'his- 
toire nationale bulgare, et qu'elle ait perduré après plusieurs changements radicaux de régime, nous incite à ouvrir l'interprétation à des considérations moins contingentes que les seules politiques culturelles ou les qualités des hommes. À titre provisoire, cette étude n'en étant qu'à ses débuts, nous conclurons en quatre points qui sont autant d'hypothèses à approfondir pour des analyses ultérieures.

- La peinture donne forme à l'imaginaire de la cité et les thèmes évoqués (les fêtes populaires, la montagne, les communautés ethniques, ...) permettent d'inscrire cet imaginaire dans des représentations plus larges qui accrochent le local à l'histoire nationale. Cependant cette construction patrimoniale de la ville se développe en sélectionnant les traits du passé : ainsi, quand on évoque Plovdiv pour des étrangers, on pense généralement aux quelques pour cent du territoire municipal qui datent de l'époque ancienne, en négligeant à la fois les constructions du début du $\mathrm{XX}^{\mathrm{e}}$ siècle et les immeubles de l'époque communiste. Même si les toiles naïves de Bojadjiev ne sont pas avares de scènes montrant la cohabitation des Bulgares avec les Turcs, les Juifs ou les Tsiganes, il faudra attendre les années 80 pour que le patrimoine ottoman soit reconnu comme appartenant de plein droit au patrimoine urbain. Et encore : le quartier de Kapana, est souvent présenté comme un quartier " d'artisans bulgares de l'époque de Renaissance bulgare ".

- Le lien entre peinture, fêtes et patrimoine alimente en permanence cet imaginaire en le ritualisant et en lui permettant de s'incarner dans la pierre et dans les paysages. Le patrimoine de Plovdiv ne peut pas être envisagé sans les animations et les usages qui permettent de l'associer à l'imaginaire de la cité. C'est, en particulier, l'esthétique de cette architecture, mise en valeur par les manifestations culturelles après avoir été portée par les politiques publiques et l'idéologie de la nation, relayée par les peintres, qui lie le présent au passé et qui donne une permanence à l'imaginaire du lieu.

- À travers le patrimoine et le site s'expriment des représentations du lieu que la peinture ou la poésie vont tenter de traduire, qui vont bien au-delà des politiques culturelles ou des intérêts économiques. Dans nos enquêtes nous avons pu remarquer combien était forte, chez les artistes, l'opinion selon laquelle le patrimoine influe sur la capacité créatrice des individus : " la ville est née depuis des siècles dans la plaine de Thrace et dès sa fondation l'art s'y est épanoui. Dès que la ville a existé, la civilisation a suivi, et la culture s'est exprimée. Peut-être est-ce dû au fait que ce lieu possède un certain magnétisme $" 49$. Ainsi le patrimoine n'est pas seulement le cadre ou l'ambiance qui construit le caractère de la ville, il est aussi considéré par certains artistes comme une force spirituelle qui soutient la création et contribue à donner son unicité à Plovdiv. 
Quand on interroge les habitants de Plodiv - et l'expérience vaut pour de nombreuses autres villes d'Europe - ils évoquent avec force ce qui fait le caractère de leur cité : "À Plovdiv, la vie coule comme du miel " dit un adage local. L'image de la ville, telle qu'elle est véhiculée par les intellectuels, par les artistes, par les responsables municipaux mais aussi par les plaquettes touristiques et sur les cartes postales est celle d'une ville d'artistes au patrimoine exceptionnel, où règne une douceur de vivre orientale et une certaine indépendance qui aurait perduré durant le communisme. Ici, il y a " une énergie positive " nous a dit l'un des principaux animateurs de la vie artistique locale ${ }^{50}$, sans qu'on sache vraiment s'il évoquait les forces telluriques ${ }^{51}$, la vitalité des artistes ou l'ambiance générale qui règne dans la ville, probablement les trois à la fois. Ce sur quoi il insistait, sans ambiguïté, c'est sur le caractère intemporel, non contingent de cette " énergie " qui devrait plus à la spécificité du site qu'aux aléas de l'histoire et qui influencerait l'action des hommes.

Ce qui est en jeu ici, c'est bien la façon dont une ville - ou tout autre lieu emblématique des activités humaines - devient un condensé de représentations et d'images qui vont l'inscrire dans l'imaginaire, cet imaginaire contribuant lui-même à définir ce qui peut (doit) être fait en matière de protection du patrimoine et d'activités artistiques et culturelles.

${ }^{51}$ Dans les années 80 , des scientifiques russes sont venus mesurer le magnétisme des roches sur lesquelles a été bâtie la vieille ville, construite dans une région à forte activité sismique. Les roches présentaient un degré de magnétisme élevé. 